\title{
Modelling intestinal inflammation and infection using 'mini-gut' organoids
}

\author{
Vivian S. W. Li (i) \\ In 2020, major advances to the understanding of gastrointestinal \\ inflammatory and infectious disease have been made using 'mini-gut' \\ organoids. Key findings include the discovery of somatic inflammatory \\ gene mutations in ulcerative colitis epithelium, a unique mutational \\ signature in colorectal cancer caused by genotoxic Escherichia coli, \\ and infection of intestinal organoids by SARS-CoV-2.
}

In 2009 , 3D organoid culture was first established when single intestinal stem cells (ISCs) were shown to grow and functionally differentiate into self-organizing 'mini-guts'. Unlike many other 3D organotypic cultures, ISC-derived organoids are purely epithelial. Owing to their unlimited expansion capacity, organoids offer unique opportunities for disease modelling, drug screening and regenerative medicine (FIG. 1). Over the past decade, a variety of living organoid biobanks have been generated from patients with genetic diseases and cancer ${ }^{2}$. These biobanks are valuable resources for better understanding of human diseases and for personalized medicine. In 2020, three studies have further strengthened our knowledge of inflammation and infection in the gastrointestinal tract using intestinal organoids.

Ulcerative colitis is characterized by chronic inflammation in the large intestine. Patients with ulcerative colitis are predisposed to colorectal cancer (CRC), suggesting that chronic exposure to the inflammatory environment can lead to somatic evolution of neoplastic clones. To understand how inflammation shapes the genetic landscape, Nanki et al. ${ }^{3}$ utilized organoids to analyse somatic mutations in the ulcerative colitis epithelium. An organoid biobank was established from 55 patients with ulcerative colitis -26 of whom had colitis-associated neoplasia - and 16 healthy individuals. Clonal organoids were expanded from single cells and subjected to whole-genome sequencing (WGS) to capture mutations at the clonal level. Analysis of single-nucleotide variants (SNVs) showed that the number of SNVs in ulcerative colitis organoids was slightly higher than in the paired control organoids derived from the uninflamed tissues of the same individuals, whereas the mutational signatures were comparable. The results suggest that inflammation leads to a moderate increase in mutation numbers in ulcerative colitis epithelium. Analysis of functional genetic mutations further revealed that recurrent truncating mutations of genes related to IL-17 signalling - NFKBIZ, PIGR and $\mathrm{ZC} 3 \mathrm{H} 12 \mathrm{~A}$ - were found exclusively in ulcerative colitis organoids, suggesting a selection for inflammation-specific mutations. CRISPR-based knockout screening of IL-17A-treated organoids resulted in enrichment of IL17RA and NFKBIZ mutations, confirming that the in vivo-selected genetic mutations confer IL-17A resistance. Interestingly, growth factor screening in these organoids revealed that deficiency of the bone morphogenetic protein (BMP) antagonist Noggin sensitized organoids to IL-17A cytotoxicity via inducible nitric oxide synthase (iNOS). As iNOS is highly expressed at the mucosal surface of ulcerative colitis tissues, the results imply that chronic inflammation in patients with ulcerative colitis activates IL-17 signalling, which triggers iNOS-dependent apoptosis at the superficial layer of the intestinal epithelium, where BMP antagonists are absent. This process subsequently drives a pattern of somatic evolution and expansion of IL-17-resistant mutant clones to protect the ulcerative colitis epithelium from IL-17-mediated apoptosis. This study elegantly demonstrates how organoids can be applied to interrogate complex somatic evolution in ulcerative colitis tissues to adapt to the chronic inflammatory microenvironment. Indeed, a parallel study, also published in 2020 , sequenced ulcerative colitis and non-ulcerative colitis crypts isolated directly from patients and similarly reported the same positive selection of mutant clones that converge on the NFKBIZ pathway ${ }^{4}$. Together, these studies suggest that organoids are reliable disease models for genomic and functional studies of human gastrointestinal diseases.

Healthy organoids can also be used for disease modelling by exposing organoids to pathogens. This year, in an insightful study, Pleguezuelos-Manzano et al. ${ }^{5}$ demonstrated how organoids can be used to probe the role of the intestinal microbiota in CRC. A genotoxic Escherichia coli strain carrying a 50-kb hybrid polyketide-nonribosomal peptide synthase operon ( $p k s)$ produces the genotoxin colibactin, which is known to induce double-strand DNA breaks. $p k s^{+}$ E. coli are present in $\sim 20 \%$ of healthy individuals and $60 \%$ of patients with CRC, suggesting a link between $p k s^{+}$E.coli and CRC development. To study this mutagenic property, a $p k s^{+} E$. coli strain and a $c l b Q$-deficient E. coli control strain that could not produce active colibactin were microinjected into the lumen of healthy human intestinal organoids. The results showed that exposure of organoids to $p k s^{+} E$. coli induced double-strand DNA breaks and interstrand crosslinks. To recapitulate long-term exposure of the pathogens in patients, single-cell-derived organoids were repeatedly injected with $p k s^{+} E$. coli over a period of 5 months, followed by WGS of the subclonal organoids. Organoids exposed to $p k s^{+}$E. coli, but not to the control strain, showed increased numbers of single-base substitutions (SBS), with a bias towards $\mathrm{T}>\mathrm{N}$ substitutions that occurred preferentially at the middle bases of ATA, ATT and TTT, as well as a small indel signature characterized by a single $\mathrm{T}$ deletion at $\mathrm{T}$ homopolymers. Importantly, interrogation of the WGS data from 5,876 human cancers generated from two independent cohorts confirmed the enrichment and co-occurrence of both $p k s$-specific SBS and indel signatures predominantly in CRCs. Notably, many driver genes, including the most commonly mutated gene,

\section{Key advances}

- Evolution of somatic inflammatory gene mutations targeting IL-17 signalling occurs during chronic tissue inflammation in patients with ulcerative colitis ${ }^{3}$.

- A distinct mutational signature caused by exposure to genotoxic colibactin-producing $\mathrm{pks}^{+}$Escherichia coli is found in a subset of colorectal cancers 5 .

- Active SARS-CoV-2 infection and replication is possible in human and bat intestinal organoids? 


\section{YEAR IN REVIEW}
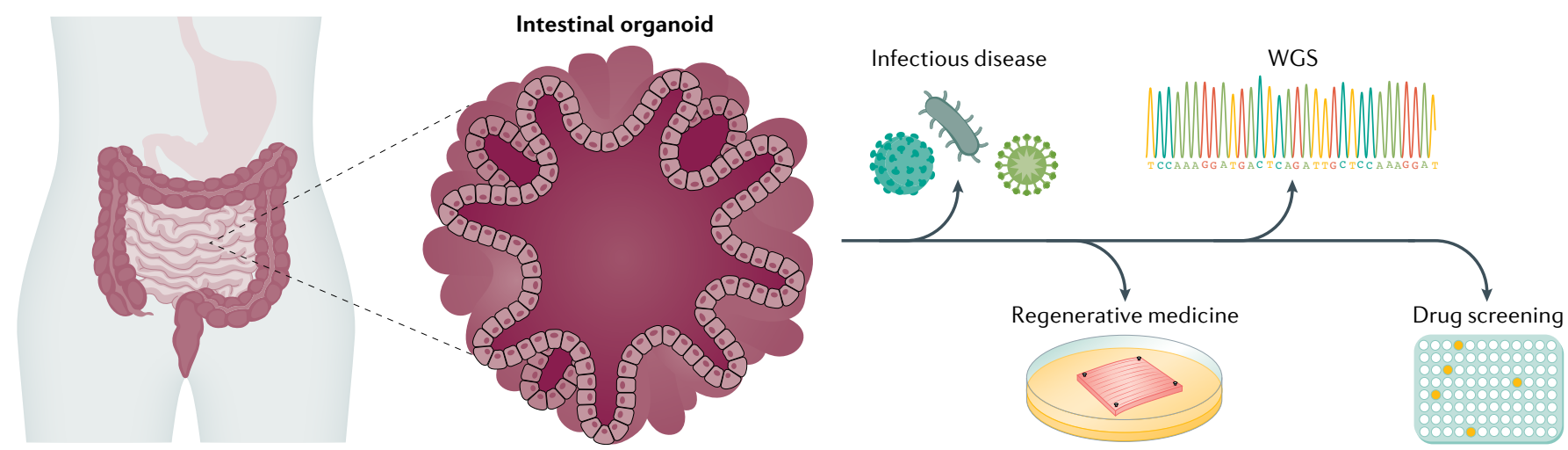

Fig. 1 | Key applications of patient-derived organoids. Human intestinal patient-derived organoids can be used for modelling infectious diseases, whole-genome sequencing (WGS), drug screening and regenerative medicine.

$A P C$, contained mutations that matched the pks-specific SBS and indel target motifs, suggesting that exposure to $p k s^{+} E$. coli might be a risk factor for CRC development. Indeed, the $p k s$-specific SBS and indel signatures identified in this study match the SBS-A and indel-A signatures reported in healthy human colon crypts in 2019 (REF. ${ }^{6}$ ), suggesting that individuals harbouring the genotoxic $p k s^{+}$ E.coli might have a higher risk of developing CRC than those who do not.

In the third study, Zhou et al. ${ }^{7}$ applied organoids to model infection of a novel coronavirus, severe acute respiratory syndrome coronavirus 2 (SARS-CoV-2), which has caused the global coronavirus disease 2019 (COVID-19) pandemic in 2020. Apart from respiratory illness, substantial numbers of patients show signs of gastrointestinal symptoms such as vomiting and diarrhoea, suggesting that the gastrointestinal tract might be an alternative route of SARS-CoV-2 infection and replication. SARS-CoV-2 was first reported in Wuhan, China, and it is believed to have originated in bats. To better understand the infection and transmission route of SARS-CoV-2, Zhou et al. established intestinal organoids derived from human as well as horseshoe bats and assessed their susceptibility to SARS-CoV-2 infection. Virus isolated from patients with COVID-19 was able to infect both human and bat organoids, leading to progressive cytopathic effects with increased viral load over time. Expression of the SARS-CoV-2 receptors angiotensinconverting enzyme 2 (ACE2) and transmembrane protease serine 2 (TMPRSS2) was markedly upregulated upon organoid differentiation, suggesting that viral infection occurs in differentiated cells. This observation is consistent with an independent study that showed infection and replication of SARS-CoV-2 in enterocytes of human intestinal organoids ${ }^{8}$. The robust infection and replication of SARS-CoV-2 in human intestinal organoids suggest that the gastrointestinal tract might be a transmission route of SARS-CoV-2. Moreover, as organoids can readily be generated from various mammalian species, the technology might become key to the study of future pandemics that are caused by species-jumping viruses.

In addition to the three studies highlighted, several other studies in 2020 have also reported exciting organoid-based findings, including the bioengineering of a scaffoldguided functional intestine using a bioreactor ${ }^{9}$ and organoids-on-a-chip ${ }^{10}$. It will be interesting to see how intestinal organoids can further be used to address more complex biological questions, such as tissue regeneration and the immune microenvironment, in the coming years.
Vivian S. W. Li (D)

Stem Cell and Cancer Biology Laboratory, The Francis Crick Institute, London, UK. e-mail:vivian.li@crick.ac.uk

https://doi.org/10.1038/s41575-020-00391-4

1. Sato, T. et al. Single Lgr5 stem cells build crypt-villus structures in vitro without a mesenchymal niche. Nature 459, 262-265 (2009).

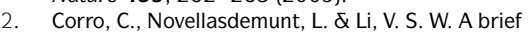 history of organoids. Am. J. Physiol. Cell Physiol. 319 C151-C165 (2020).

3. Nanki, K. et al. Somatic inflammatory gene mutations in human ulcerative colitis epithelium. Nature 577 , 254-259 (2020).

4. Kakiuchi, N. et al. Frequent mutations that converge on the NFKBIZ pathway in ulcerative colitis. Nature 577, 260-265 (2020).

5. Pleguezuelos-Manzano, C. et al. Mutational signature in colorectal cancer caused by genotoxic pks(+) E. coli. Nature 580, 269-273 (2020).

6. Lee-Six, H. et al. The landscape of somatic mutation in normal colorectal epithelial cells. Nature $\mathbf{5 7 4}$ 532-537 (2019).

7. Zhou, J. et al. Infection of bat and human intestinal organoids by SARS-CoV-2. Nat. Med. 26, 1077-1083 (2020).

8. Lamers, M. M. et al. SARS-CoV-2 productively infects human gut enterocytes. Science 369, 50-54 (2020)

9. Meran, L. et al. Engineering transplantable jejunal mucosal grafts using patient-derived organoids from children with intestinal failure. Nat. Med. 26, 1593-1601 (2020).

10. Nikolaev, M et al. Homeostatic mini-intestines through scaffold-guided organoid morphogenesis. Nature 585, 574-578 (2020).

\section{Acknowledgements}

The laboratory of V.S.W.L. is funded by the Francis Crick Institute, which receives its core funding from Cancer Research UK (FC001105), the UK Medical Research Council (FC001105) and the Wellcome Trust (FC001105).

\section{Competing interests}

The authors declare no competing interests. 